\title{
SPORTS-RELATED INJURIES IN CHINESE PARALYMPIC ATHLETES
}

\author{
Chunxiao Li*, Lawrence T. Lam \\ The Hong Kong Institute of Education \\ Yandan Wu \\ Fujian Normal University
}

Chunxiao Li and Lawrence T. Lam are with the Department of Health and Physical Education, The Hong Kong Institute of Education, Hong Kong, China. Yandan Wu is with the School of Physical Education and Sports Science, Fujian Normal University, Fuzhou, China.

*Corresponding author: Chunxiao Li, Department of Health and Physical Education, The Hong Kong Institute of Education, D2-2/F-24, 10 Lo Ping Road, Tai Po, New Territories, Hong Kong, E-mail: cxlilee@gmail.com, Tel: +852 29488913, Fax: +852 29487848

\begin{abstract}
This study aimed to explore the patterns, characteristics, and perceived risk factors of sports-related injuries among Paralympic athletes. One hundred and sixty Chinese athletes with either physical disabilities or visual impairments participating at a province-level Paralympic Games were recruited. Data on the occurrence of injuries during training and competitions were collected using a self-reported injury form. Of the 160 respondents, 82 (51\%) reported a sports-related injury with track and field being the most commonly reported place of injury and lower limbs $(77 \%)$ the most reported body area in young athletes with disabilities. Tried and not in condition (50\%), decline in muscle coordination (35\%), overtraining (39\%), anxiety/overexcitement (39\%), weather (46\%), and lack of awareness for self-protection (43\%) were nominated as main risk factors of injuries. Anxiety/overexcitement was perceived as a major cause resulting in moderate and severe injuries. It was concluded that the prevalence of injury among Chinese Paralympic athletes involving in the province-level Paralympic Games was relatively high. Risk factors especially for training volume, anxiety/overexcitement, weather, and self-protection awareness should be considered when planning injury prevention strategies.
\end{abstract}

\section{Keywords: Paralympics, epidemiology, Chinese, sport}

Sports participation is an important way for people with disabilities to maintain and improve their physical and psychological health (Blauwet \& Willick, 2012). There has been an increasing participations in the Paralympic Games in different countries in recent years (Blauwet \& Willick, 2012; Webborn,Willick, \& Emery, 2012). In 2012, it was witnessed that over 4000 athletes with physical, visual, and intellectual impairments participated in the London Paralympic Games. Sports participation, however, increases the risk of injuries. Sports-related injuries may lower sports performance and result in long-term morbidity and mortality (Ljungqvist et al., 2009). Sports-related injuries can also cause economic burdens for the society such as reduced work capacity and increased costs of medical treatment (Ljungqvist et al., 2009). These negative consequences will be more serve among athletes with disabilities compared with those who without (Fagher \& Lexell, 2014). For example, sports-related injuries may further impair activities of daily living in an athlete with physical disabilities. To this end, epidemiologic studies of sports-related injuries for athletes with disabilities are important.

Understanding sports-related injuries among athletes with disabilities is not new. For example, Ferrara and Buckley (1996) began a project in 1990 introducing an injury reporting system to identify the injury patterns and trends for athletes with disabilities from 
different disabled sports associations (e.g., Special Olympics). Ferrara and colleagues (2000) conducted a longitudinal study to investigate the number and type of injuries occurred to athletes with disabilities between 1990 and 1996. They found that the majority of the injuries were musculoskeletal and were mainly located on the spine, shoulder, and lower limb.

Despite a relatively long history of the inquiry line and a few significant studies (Ferrara \& Buckley, 1996; Ferrara et al., 2000), little research has been done to understand sports-related injuries among athletes with disabilities (Fagher \& Lexell, 2014). Recent review studies summarized that the majority of early studies used self-report data, a high prevalence of overuse injuries, lower extremity injuries were more common in walking athletes, shoulder injuries were mostly reported in wheelchair athletes, and injury incident rates in athletes with disabilities were comparable to able-bodied athletes, (Fagher \& Lexell, 2014; Webborn \& Emery, 2014). Studies also highlighted the need to investigate injury severity, risk factors, and prevention strategies in Paralympic athletes (Fagher \& Lexell, 2014; Webborn \& Emery, 2014). Moreover, very few studies, to date, reported sports-related injuries among athletes participating in a larger scale and at lower levels of games such as at the province-level Paralympic Games. Their injury pattern may be different from those athletes who are competing at higher competition levels.

This study aims to investigate the patterns and characteristics of sports-related injuries in Chinese Paralympic athletes with either physical disabilities or visual impairments who participated in a province-level Paralympic Games. It also aims to examine the perceived risk factors of sports-related injuries to provide better information in developing injury prevention strategies for this particular population.

\section{Methods}

This was a cross-sectional population-based study of sports-related injuries in Chinese athletes attending at the 7th Paralympic Games in Fujian Province. Ethical approval was obtained from the principle investigator's university. The Fujian Paralympic Committee also gave permission for access to athletes. All athletes who participated in the study signed written consent forms.

\section{Participants}

The study sample of this research consisted of Paralympic athletes $(N=241$, male $=184$, female $=57$ ) attending the Games. They suffered from either physical disabilities $(n=195)$ or visual impairments $(n=46)$ with a mean age of 28.5 years $(\mathrm{SD}=11.2)$. All athletes participating in the Games were invited to participate in this study.

Table 1

Demographics and other Characteristics of Athletes of the Paralympic Games $(n=160)$

\begin{tabular}{lc}
\hline Characteristics & $\begin{array}{c}\text { Valid Frequency } \\
(\%) \text { or Mean (SD) }\end{array}$ \\
\hline Gender & $122(78 \%)$ \\
Male & $35(22 \%)$ \\
Female & $28.1(9.9)$ \\
Age & \\
Disability type & $20(16 \%)$ \\
Visual impairment & $108(84 \%)$ \\
Physical impairment & \\
Sport & $15(10 \%)$ \\
Badminton & $15(10 \%)$ \\
Dart & $22(14 \%)$ \\
Swimming & $13(8 \%)$ \\
Table tennis & $89(58 \%)$ \\
Track \& field & $3.3(4.4)$ \\
No. of years of training & $4.8(2.1)$ \\
No. of training days /week & $3.1(1.7)$ \\
Training hours /day & \\
Highest Competition Level & $7(7 \%)$ \\
International & $30(29 \%)$ \\
National & $76(64 \%)$ \\
Province & \\
Injury & $82(51 \%)$ \\
Yes & $76(49 \%)$ \\
No & \\
Injury severity & $5(6 \%)$ \\
Slight & $15(19 \%)$ \\
Minimal & $45(55 \%)$ \\
Mild & \\
Moderate &
\end{tabular}


Severe $1(1 \%)$

\section{Instrument and procedures}

"Injury" was defined as any sports-related musculoskeletal complaint that resulted in an athlete to stop, limit, or modify participation for one or more days (Ferrara \& Buckley, 1996; Ferrara, Buckley, McCann, Limbird, Powell, \& Robl, 1992). With regards to the location of injury, the body was divided into four segments: head (head/face), upper limbs (shoulder, upper arm/forearm, elbow, wrist, hand/digits), trunk (neck/cervical spine, chest, abdomen, waist, and back), and lower limb (hip/groin, thigh, knee, lower leg/Achilles tendon, ankle, foot/toe).

A study instrument (i.e., an injury report form) was developed based on the earlier studies within this field (Morato, Bilzon, \& Duarte, 2012; Willick et al., 2013). The following information was collected through the form: open-ended questions (i.e., gender, age, disability type, sports type, number of years of sports participation, number of training days per week, training hours each day, highest competition level) and multiple-choice items (i.e., injury severity, when injury occurred, type of injury, injured body part, and perceived risk factors of injury). Injuries occurred to athletes during training and competitions in the previous 12 months were counted. A recurrent injury (the same injury that previously sustained) was counted once.

The whole study population was invited by the Fujian Paralympic Committee to participate in this study during the Games (pre-competition). Upon obtaining participants' informed consent, the injury report forms were distributed to athletes with physical disabilities in quite meeting rooms under researchers' supervision. For athletes with visual impairments, the data was collected through a face-to-face interview by researchers. Participants were informed that there was no correct or wrong answer for the survey. It took approximately 10 minutes to complete the survey.

\section{Data analysis}

Data were analyzed using SPSS $21.0^{\circledR}$. Missing values were not imputed, and available information was analyzed and reported. Descriptive statistics (i.e., frequency, percentage, mean, standard deviation) were used to determine the collected information. Chi-square tests were applied to compare the gender, highest level of competition, sports type, and disability type differences on the injured and non-injured groups. The same test was also used to compare differences of injury severity by perceived risk factors of injury. Finally, an independent t-test was employed to examine whether there was an age difference between the injured and non-injured groups. The significant level was set at .05 .

\section{Results}

One hundred and sixty Paralympic athletes involved in the Games responded the survey form with a response rate of $66 \%$. Comparisons between respondents and non-respondents on age, sex, and type of disability indicated no significant group differences. The demographics and other characteristics of the participants were summarized in Table 1. In brief, the majority of respondents were males $(n=122,78 \%)$ and the average age of the sample was 28.1 years (SD = 9.9). Among those who provided information on their disabilities, the majority were athletes with physical disabilities (84\%). Half $(n=82,51 \%)$ of the respondents reported that they had a sports-related injury and $20 \%$ $(n=16)$ of them perceived their injuries to be moderate to severe (Table 1). Comparisons on the demographics between the injured and non-injured groups suggested no significant differences between sex, highest level of competition and disability type. However, there was a significant difference of sports type on the injured and non-injured groups with the majority of the injured athletes involving in track and field $(n=60,87 \% ; p<$ $0.001)$. In addition, the injured athletes were significantly younger than the non-injured athletes with mean ages of $26.2(\mathrm{SD}=9.3)$ and 
$30.1(\mathrm{SD}=10.1)$ respectively $\left(t_{[155]}=-2.51, p\right.$ $=0.013)$.

The injury related information was tabulated in Table 2. The most common activity involved while injury occurred was during training. Half $(n=42,51 \%)$ of the injured reported that they were injured during a specialty training session with torn muscle being the most common type of injury ( $n=49$, $60 \%)$ and followed by strain of a tendon $(n=$ $31,38 \%)$. In terms of injured body regions, the majority had experienced an injury to their lower limbs $(n=63,77 \%)$ with thigh being the most common body part $(n=29,35 \%)$.

For the perceived risk factors of injury (Table 3), tired and not in condition was nominated as the most common reason for body function $(n=41,50 \%)$. More than one-third of the injured participants $(n=29$, $35 \%$ ) thought that decline in muscle coordination was also a physical factor for the injury. The most common reason for injuries in the area of organization of training was overload volume $(n=32,39 \%)$ and followed by the continuation of training despite injuries or illness $(n=24,29 \%)$. Anxiety/overexcitement was nominated as the most common psychological factor of injuries by 32 athletes $(39 \%)$ and weather was the most common environmental factor by 38 respondents $(46 \%)$. In terms of the personal reasons, lack of awareness for self-protection was identified as the most common reason of injuries $(n=35,43 \%)$.

When the main perceived risk factor of injury of each category were compared between severity levels of injury, the results indicated that less athletes with severe/moderate injuries nominated tired and not in condition as the reason $(25 \%)$ than those who suffered only mild injuries $(58 \%)$ ( $p$ $=0.019)$. On the other hand, athletes with severe/moderate injuries were more likely to nominate anxiety or overexcitement as the reason of injury $(63 \%)$ in comparison to those with mild injuries $(35 \%)(p=0.045)$. No significant differences were found in other main factors of injury by severity levels of injury.
Table 2

Details on the Injuries $(n=82)$

\begin{tabular}{|c|c|}
\hline Category & Frequency $(\%) *$ \\
\hline \multicolumn{2}{|l|}{ When Injury Occurred } \\
\hline During specialty training & $42(51 \%)$ \\
\hline During physical training & $28(34 \%)$ \\
\hline During skills training & $27(33 \%)$ \\
\hline During competition & $21(26 \%)$ \\
\hline \multicolumn{2}{|l|}{ Type of Injury } \\
\hline Muscle torn & $49(60 \%)$ \\
\hline Tendon strain & $31(38 \%)$ \\
\hline Dislocation of joints & $4(5 \%)$ \\
\hline Bone fractures & $6(7 \%)$ \\
\hline Others & $10(12 \%)$ \\
\hline \multicolumn{2}{|l|}{ Body Part } \\
\hline Head/face & $3(4 \%)$ \\
\hline Neck/cervical spine & $10(12 \%)$ \\
\hline Shoulder & $19(23 \%)$ \\
\hline Upper arm/forearm & $20(24 \%)$ \\
\hline Elbow & $13(16 \%)$ \\
\hline Wrist & $10(12 \%)$ \\
\hline Hand/digits & $8(10 \%)$ \\
\hline Chest & $3(4 \%)$ \\
\hline Abdomen & $4(5 \%)$ \\
\hline Waist & $33(40 \%)$ \\
\hline Back & $6(7 \%)$ \\
\hline Hip/groin & $1(1 \%)$ \\
\hline Thigh & $29(35 \%)$ \\
\hline Knee & $22(27 \%)$ \\
\hline Lower leg/Achilles tendon & $26(32 \%)$ \\
\hline Ankle & $18(22 \%)$ \\
\hline Foot/toe & $13(16 \%)$ \\
\hline \multicolumn{2}{|l|}{ Body Segment } \\
\hline Head & $3(4 \%)$ \\
\hline Upper limb & $31(38 \%)$ \\
\hline Trunk & $36(44 \%)$ \\
\hline Lower limb & $63(77 \%)$ \\
\hline
\end{tabular}

Note. *Multiple responses

Table 3

Perceived Reasons of Injury $(n=82)$

\begin{tabular}{lc}
\hline Possible Causal Factors & Frequency $(\%)^{*}$ \\
\hline Body Function & $16(20 \%)$ \\
History of injury & $41(50 \%)$ \\
Tired, not in condition & $2(2 \%)$ \\
Having period & $20(24 \%)$ \\
Decline of body fitness & \\
Physical Factor & $15(18 \%)$ \\
Unbalance of muscle & $29(35 \%)$ \\
development & $16(20 \%)$ \\
Decline of muscle coordination & \\
Decline of muscle strength & $6(7 \%)$ \\
Muscular strength growing too & $18(22 \%)$ \\
fast & $15(18 \%)$ \\
Decline of flexibility & $13(16 \%)$ \\
Decline of endurance & \\
Decline of speed & $32(39 \%)$ \\
Organization of Training & \\
Overload volume &
\end{tabular}




$\begin{array}{lc}\text { Continue training with } & \\ \text { injuries/illness } & 24(29 \%) \\ \text { Insufficient warm-up } & 23(28 \%) \\ \text { Wrong sports techniques } & 19(23 \%) \\ \text { Training programs suddenly } & \\ \text { changed } & 10(12 \%) \\ \quad \text { Improper protection measures } & 10(12 \%) \\ \text { Psychological Factor } & 32(39 \%) \\ \text { Anxiety or overexcitement } & 17(21 \%) \\ \text { Inattention or too much attention } & \\ \text { Lack of self-protection } & 24(29 \%) \\ \text { awareness } & \\ \text { Environmental Factor } & 38(46 \%) \\ \quad \text { Weather } & 14(17 \%) \\ \text { Field } & 3(4 \%) \\ \text { Equipment } & 4(5 \%) \\ \text { Others } & \\ \text { Self-Management } & 35(43 \%) \\ \text { Low self-protection awareness } & \\ \text { Low effective prevention } & 26(32 \%) \\ \text { measures } & 10(12 \%) \\ \text { Irregular rest patterns } & \end{array}$

Note. *Multiple responses

\section{Discussion}

This epidemiological study investigated the patterns, characteristics, and perceived risk factors of sports-related injury among Chinese athletes with either physical disabilities or visual impairments participating in the province-level Paralympic Games. There are several important findings in the current research. Although relatively high injury prevalence was found $(51 \%)$, the majority of these injuries were not severe in nature $(80 \%)$. The result is in line with prior reports (Ferrara \& Peterson, 2000; Webborn, Willick, \& Reeser, 2006).

An interesting result is that the reported injury did not depend on disability categories even the disability itself could be considered as an intrinsic risk factor for sports-related injury (van Mechelen, Hlobil, \& Kemper, 1992). This is possibly due to our participants were overly represented by athletes with physical disabilities (84\%). Instead of disability categories, sports types could be considered as an intrinsic risk factor. The current study showed that track and field athletes sustained more injuries than those involving in other events. That means track and field is a higher risk event compared with other sports such as badminton and swimming
(Willick et al., 2013). Another identified intrinsic risk factor related to sports-related injuries is age. Younger athletes $\left(M_{\text {age }}=26.2\right)$ tended to report an injury than older ones $\left(M_{\mathrm{age}}=30.1\right)$. One possible explanation is that the older athletes might have higher self-protection awareness than the younger age-group. Contextual factors such as the knowledge level and experience of coaches may also explain the difference. The precise reason requires further research.

According to previous research (Ferrara \& Peterson, 2000; Ferrara et al., 2000; Magno et al., 2012), soft tissue injuries were commonly reported among athletes with disabilities. The similar pattern was detected in this study, where sprains and strains were more common than fractures and dislocations. Regarding injured body regions, lower extremity was most common (77\%) mainly because most participants were not wheelchair users based on our observation. This finding is consistent with earlier studies showing that lower extremity injuries were more common in walking athletes (Chung et al., 2012; Magno et al., 2012; Magno, Winckler, Costa, Bilzon, \& Duarte, 2013; Patatoukas et al., 2011).

As expected, most injuries occurred during training rather than competitions because the athletes generally spend more time on training. Overtraining, however, was reported as an extrinsic risk factor of injury. Similarly, tired and not in condition was nominated by half of the injured participants as an intrinsic causal factor. These may explain why the prevalence of overuse injuries is considerably high in athletes with disabilities (Fagher \& Lexell, 2014). These also suggest the importance of monitoring the training volume and recovery for injury prevention among this special population (Bernardi et al., 2003).

A large proportion of the injured athletes nominated anxiety/overexcitement as well as low self-protection awareness as other intrinsic risk factors, indicating the need to prepare athletes psychologically for training and competitions. There is also a need to educate athletes with disabilities to raise their 
self-protection awareness. On the other hand, an important extrinsic risk factor discovered in this study was weather. Past research has shown that weather is a risk factor of sports-related injury among athletes without disabilities (Castellani \& Young, 2012; Lee \& Garraway, 2000; Ruedl, Fink, Schranz, Sommersacher, Nachbauer, \& Burtscher, 2012).

The current survey has three major strengths. First, this is the first study to date reporting on sports-related injuries in athletes attending the province-level Paralympic Games. The study findings can be used as a baseline for follow-up research. Second, a representative sample with an acceptable compliance rate $(68 \%)$ was recruited. This increases the generalizability of the study findings. Third, a novel contribution of this research is to understand the perceived risk factors of injury by athletes with disabilities. Meanwhile, several limitations face this study. One limitation is that self-report data that did not include a confirmed medical diagnosis may bias the results. Another limitation concerns a relatively broad overview of injury patterns without carefully considering classification systems, disability categories, and other personal characteristics. This also makes the comparisons between earlier study findings and the current results difficult. Yet, it is worthy to note that there are over 110 classifications across Paralympic sports, which makes more refined and detailed analyses of data infeasible especially when the sample size is small (Patatoukas et al., 2011).

In summary, Chinese athletes participating in the province-level Paralympic Games sustain relatively high injury prevalence. Most of the reported injuries are located in the lower extremity and reported by track and field athletes. Risk factors such as training volume, anxiety/overexcitement, weather, and self-protection awareness should be considered for injury prevention.

\section{References}

Bernardi, M., Castellano, V., Ferrara, M. S., Sbriccoli, P., Sera, F., \& Marchetti, M.
(2003). Muscle pain in athletes with locomotor disability. Medicine \& Science in Sports \& Exercise, 35, 199-206.

Blauwet, C., \& Willick, S. E. (2012). The Paralympic movement: Using sports to promote health, disability rights, and social integration for athletes with disabilities. $P M \& R, 4,851-856$.

Castellani, J. W., \& Young, A. J. (2012). Health and performance challenges during sports training and competition in cold weather. British Journal of Sports Medicine, 46, 788-791.

Chung, W. M., Yeung, S., Wong, A. Y. L., Lam, I. F., Tse, P. T. F., Daswani, D., \& Lee, R. (2012). Musculoskeletal injuries in elite able-bodied and wheelchair foil fencers-A pilot study. Clinical Journal of Sport Medicine, 22, 278-280.

Fagher, K., \& Lexell, J. (2014). Sports-related injuries in athletes with disabilities. Scandinavian journal of Medicine \& Science in Sports, 24, e320-e331.

Ferrara, M. S., \& Buckley, W. E. (1996). Athletes with disabilities injury registry. Adapted Physical Activity Quarterly, 13, 50-60.

Ferrara, M. S., \& Peterson, C. L. (2000). Injuries to athletes with disabilities. Sports Medicine, 30, 137-143.

Ferrara, M. S., Buckley, W. E., McCann, B. C., Limbird, T. J., Powell, J. W., \& Robl, R. O. B. I. N. (1992). The injury experience of the competitive athlete with a disability: Prevention implications. Medicine \& Science in Sports \& Exercise, 24, 184-188.

Ferrara, M. S., Palutsis, G. R., Snouse, S., \& Davis, R. W. (2000). A longitudinal study of injuries to athletes with disabilities. International Journal of Sports Medicine, 21, 221-224.

Lee, A. J., \& Garraway, W. M. (2000). The influence of environmental factors on rugby football injuries. Journal of Sports Sciences, 18, 91-95.

Ljungqvist, A., Jenoure, P., Engebretsen, L., Alonso, J. M., Bahr, R., Clough, A., \& Thill, C. (2009). The International 
Olympic Committee (IOC) Consensus Statement on periodic health evaluation of elite athletes March 2009. British Journal of Sports Medicine, 43, 631-643.

Magno, E. S. M., Winckler, C., Costa, E. S. A., Bilzon, J., \& Duarte, E. (2013). Sports injuries in Paralympic track and field athletes with visual impairment. Medicine \& Science in Sports \& Exercise, 45, 908-913.

Morato, M. P., Bilzon, J. L., \& Duarte, E. (2013). Sports injuries in Brazilian blind footballers. International Journal of Sports Medicine, 34, 239-243.

Patatoukas, D., Farmakides, A., Aggeli, V., Fotaki, S., Tsibidakis, H., Mavrogenis, A., \& Papagelopoulos, P. (2011). Disability-related injuries in athletes with disabilities. Folia Medica, 53, 40-46.

Ruedl, G., Fink, C., Schranz, A., Sommersacher, R., Nachbauer, W., \& Burtscher, M. (2012). Impact of environmental factors on knee injuries in male and female recreational skiers. Scandinavian Journal of Medicine \& Science in Sports, 22, 185-189.

van Mechelen, W., Hlobil, H., \& Kemper, H. C. (1992). Incidence, severity, aetiology and prevention of sports injuries. Sports Medicine, 14, 82-99

Webborn, N., \& Emery, C. (2014). Descriptive epidemiology of Paralympic sports injuries. $P M \& R, 6, \mathrm{~S} 18-\mathrm{S} 22$.

Webborn, N., Willick, S., \& Emery, C. A. (2012). The injury experience at the 2010 Winter Paralympic Games. Clinical Journal of Sport Medicine, 22, 3-9.

Webborn, N., Willick, S., \& Reeser, J. C. (2006). Injuries among disabled athletes during the 2002 Winter Paralympic Games. Medicine \& Science in Sports \& Exercise, 38, 811-815.

Willick, S. E., Webborn, N., Emery, C., Blauwet, C. A., Pit-Grosheide, P., Stomphorst, J., \& Schwellnus, M. (2013). The epidemiology of injuries at the London 2012 Paralympic Games. British Journal of Sports Medicine, 47, 426-432. 